\title{
Sharply Orthocomplete Effect Algebras
}

\author{
M. Kalina, J. Paseka, Z. Riečanová
}

\begin{abstract}
Special types of effect algebras $E$ called sharply dominating and S-dominating were introduced by S. Gudder in [7, 8]. We prove statements about connections between sharp orthocompleteness, sharp dominancy and completeness of $E$. Namely we prove that in every sharply orthocomplete S-dominating effect algebra $E$ the set of sharp elements and the center of $E$ are complete lattices bifull in $E$. If an Archimedean atomic lattice effect algebra $E$ is sharply orthocomplete then it is complete.
\end{abstract}

Keywords: effect algebra, sharp element, central element, block, sharply dominating, S-dominating, sharply orthocomplete.

\section{Introduction}

An algebraic structure called an effect algebra was introduced by D. J. Foulis and M. K. Bennett (1994). The advantage of effect algebras is that they provide a mechanism for studying quantum effects, or more generally, in non-classical probability theory their elements represent events that may be unsharp or pairwise non-compatible. Lattice effect algebras are in some sense a nearest common generalization of orthomodular lattices [13] that may include noncompatible pairs of elements, and MV-algebras [3] that may include unsharp elements. More precisely, a lattice effect algebra $E$ is an orthomodular lattice iff every element of $E$ is sharp (i.e., $x$ and "non $x$ " are disjoint) and it is an MV-effect algebra iff every pair of elements of $E$ is compatible. Moreover, in every lattice effect algebra $E$ the set of sharp elements is an orthomodular lattice ([10]), and $E$ is a union of its blocks (i.e., maximal subsets of pairwise compatible elements that are MV-effect algebras (see [21])). Thus a lattice effect algebra $E$ is a Boolean algebra iff every pair of elements is compatible and every element of $E$ is sharp.

However, non-lattice ordered effect algebra $E$ is so general that its set $S(E)$ of sharp elements may form neither an orthomodular lattice nor any regular algebraic structure. S. Gudder (see [7, 8]) introduced special types of effect algebras $E$ called sharply dominating effect algebras, whose set $S(E)$ of sharp elements forms an orthoalgebra and also socalled S-dominating effect algebras, whose set $S(E)$ of sharp elements forms an orthomodular lattice. In [7], S. Gudder showed that a standard Hilbert space effect algebra $\mathcal{E}(H)$ of bounded operators on a Hilbert space $H$ between zero and identity operators (with partially defined usual operation + ) is S- dominating. Hence S-dominating effect algebras may be useful abstract models for sets of quantum effects in physical systems.

We study these two special kinds of effect algebras. We show properties of some remarkable subeffect algebras of such effect algebras $E$ satisfying the condition that $E$ is sharply orthocomplete. Namely properties of their blocks, sets of sharp elements and their centers. It is worth noting that it was proved in [11] that there are even Archimedean atomic MVeffect algebras which are not sharply dominating, hence they are not S-dominating.

\section{Basic definitions and some known facts}

Definition 1 ([4]) A partial algebra $(E ; \oplus, 0,1)$ is called an effect algebra if 0, 1 are two distinct elements and $\oplus$ is a partially defined binary operation on $E$ which satisfy the following conditions for any $x, y, z \in E$ :

(Ei) $\quad x \oplus y=y \oplus x$ if $x \oplus y$ is defined,

(Eii) $\quad(x \oplus y) \oplus z=x \oplus(y \oplus z)$ if one side is defined,

(Eiii) for every $x \in E$ there exists a unique $y \in E$ such that $x \oplus y=1$ (we put $x^{\prime}=y$ ),

(Eiv) if $1 \oplus x$ is defined then $x=0$.

We often denote the effect algebra $(E ; \oplus, 0,1)$ briefly by $E$. On every effect algebra $E$ the partial order $\leq$ and a partial binary operation $\ominus$ can be introduced as follows:

$x \leq y$ and $y \ominus x=z$ iff $x \oplus z$ is defined and $x \oplus z=y$.

If $E$ with the defined partial order is a lattice (a complete lattice) then $(E ; \oplus, 0,1)$ is called a lattice effect algebra (a complete lattice effect algebra).

This paper is a contribution to the Proceedings of the 6-th Microconference "Analytic and Algebraic Methods VI". 
Definition 2 Let $E$ be an effect algebra. Then $Q \subseteq$ $E$ is called a sub-effect algebra of $E$ if

(i) $1 \in Q$,

(ii) if out of elements $x, y, z \in E$ with $x \oplus y=z$ two are in $Q$, then $x, y, z \in Q$.

If $E$ is a lattice effect algebra and $Q$ is a sub-lattice and a sub-effect algebra of $E$ then $Q$ is called a sublattice effect algebra of $E$.

Note that a sub-effect algebra $Q$ (sub-lattice effect algebra $Q$ ) of an effect algebra $E$ (of a lattice effect algebra $E$ ) with inherited operation $\oplus$ is an effect algebra (lattice effect algebra) in its own right.

For an element $x$ of an effect algebra $E$ we write $\operatorname{ord}(x)=\infty$ if $n x=x \oplus x \oplus \ldots \oplus x$ (n-times) exists for every positive integer $n$ and we write ord $(x)=n_{x}$ if $n_{x}$ is the greatest positive integer such that $n_{x} x$ exists in $E$. An effect algebra $E$ is Archimedean if ord $(x)<\infty$ for all $x \in E$.

A minimal nonzero element of an effect algebra $E$ is called an atom and $E$ is called atomic if under every nonzero element of $E$ there is an atom.

For a poset $P$ and its subposet $Q \subseteq P$ we denote, for all $X \subseteq Q$, by $\bigvee_{Q} X$ the join of the subset $X$ in the poset $Q$ whenever it exists.

We say that a finite system $F=\left(x_{k}\right)_{k=1}^{n}$ of not necessarily different elements of an effect algebra $(E ; \oplus, 0,1)$ is orthogonal if $x_{1} \oplus x_{2} \oplus \ldots \oplus x_{n}$ (written $\bigoplus_{k=1}^{n} x_{k}$ or $\bigoplus F$ ) exists in $E$. Here we define $x_{1} \oplus x_{2} \oplus \ldots \oplus x_{n}=\left(x_{1} \oplus x_{2} \oplus \ldots \oplus x_{n-1}\right) \oplus x_{n}$ supposing that $\bigoplus_{k=1}^{n-1} x_{k}$ is defined and $\bigoplus_{k=1}^{n-1} x_{k} \leq x_{n}^{\prime}$. We also define $\bigoplus \oslash=0$. An arbitrary system $G=\left(x_{\kappa}\right)_{\kappa \in H}$ of not necessarily different elements of $E$ is called orthogonal if $\bigoplus K$ exists for every finite $K \subseteq G$. We say that for an orthogonal system $G=\left(x_{\kappa}\right)_{\kappa \in H}$ the element $\bigoplus G$ (more precisely $\bigoplus_{E} G$ ) exists iff $\bigvee\{\bigoplus K \mid K \subseteq G$ is finite $\}$ exists in $E$ and then we put $\bigoplus G=\bigvee\{\bigoplus K \mid K \subseteq G$ is finite $\}$. (Here we write $G_{1} \subseteq G$ iff there is $H_{1} \subseteq H$ such that $\left.G_{1}=\left(x_{\kappa}\right)_{\kappa \in H_{1}}\right)$.

We call an effect algebra $E$ orthocomplete [9] if every orthogonal system $G=\left(x_{\kappa}\right)_{\kappa \in H}$ of elements of $E$ has the sum $\bigoplus G$. It is known that every orthocomplete Archimedean lattice effect algebra $E$ is a complete lattice (see [22, Theorem 2.6]).

Recall that elements $x, y$ of a lattice effect algebra $E$ are called compatible (written $x \leftrightarrow y$ ) iff $x \vee y=x \oplus(y \ominus(x \wedge y))$ (see [15]). $P \subseteq E$ is a set of pairwise compatible elements if $x \leftrightarrow y$ for all $x, y \in P . \quad M \subseteq E$ is called a block of $E$ iff $M$ is a maximal subset of pairwise compatible elements. Every block of a lattice effect algebra $E$ is a sub-effect algebra and a sub-lattice of $E$ and $E$ is a union of its blocks (see [21]). A lattice effect algebra with a unique block is called an $M V$-effect algebra. Every block of a lattice effect algebra is an MV-effect algebra in its own right.

An element $w$ of an effect algebra $E$ is called sharp (see $[7,8])$ if $w \wedge w^{\prime}=0$.

Definition 3 ([7,8]) An effect algebra $E$ is called sharply dominating if for every $x \in E$ there exists $\widehat{x} \in S(E)$ such that

$$
\begin{aligned}
\widehat{x}= & \bigwedge_{E}\{w \in S(E) \mid x \leq w\}= \\
& \bigwedge_{S(E)}\{w \in S(E) \mid x \leq w\} .
\end{aligned}
$$

Note that clearly $E$ is sharply dominating iff for every $x \in E$ there exists $\widetilde{x} \in S(E)$ such that

$$
\begin{aligned}
\widetilde{x}= & \bigvee_{E}\{w \in S(E) \mid x \geq w\}= \\
& \bigvee_{S(E)}\{w \in S(E) \mid x \geq w\} .
\end{aligned}
$$

A sharply dominating effect algebra $E$ is called S-dominating [8] if $x \wedge w$ exists for every $x \in E$, $w \in S(E)$.

It is a well known fact that in every S-dominating effect algebra $E$ the subset $S(E)=\left\{w \in E \mid w \wedge w^{\prime}=\right.$ $0\}$ of sharp elements of $E$ is a sub-effect algebra of $E$ being an orthomodular lattice (see [8, Theorem 2.6]). Moreover if for $D \subseteq S(E)$ the element $\bigvee D$ exists then $\bigvee_{E} D \in S(E)$ hence $\bigvee_{S(E)} D=\bigvee_{E} D$. We say that $S(E)$ is a full sublattice of $E$ (see [10]).

Let $G$ be a sub-effect algebra of an effect algebra $E$. We say that $G$ is bifull in $E$, if, for any $D \subseteq G$ the element $\bigvee_{G} D$ exists iff the element $\bigvee_{E} D$ exists and they are equal. Clearly, any bifull sub-effect algebra of $E$ is full but not conversely (see [12]).

The notion of a central element of an effect algebra $E$ was introduced by Greechie-FoulisPulmannová [6]. An element $c \in E$ is called central (see [18]) iff for every $x \in E$ there exist $x \wedge c$ and $x \wedge c^{\prime}$ and $x=(x \wedge c) \vee\left(x \wedge c^{\prime}\right)$. The center $C(E)$ of $E$ is the set of all central elements of $E$. Moreover, $C(E)$ is a Boolean algebra, see [6]. If $E$ is a lattice effect algebra then $z \in E$ is central iff $z \wedge z^{\prime}=0$ and $z \leftrightarrow x$ for all $x \in E$, see [19]. Thus in a lattice effect algebra $E, C(E)=B(E) \cap S(E)$, where $B(E)=\bigcap\{M \subseteq E \mid M$ is a block of $E\}$ is called the compatibility center of $E$. 
An effect algebra $E$ is called centrally dominating (see also [5] for the notion central cover) if for every $x \in E$ there exists $c_{x} \in C(E)$ such that

$$
\begin{array}{r}
c_{x}=\bigwedge_{E}\{c \in C(E) \mid x \leq c\}= \\
\bigwedge_{C(E)}\{c \in C(E) \mid x \leq c\} .
\end{array}
$$

An element $a$ of a lattice $L$ is called compact iff, for any $D \subseteq L, a \leq \bigvee D$ implies $a \leq \bigvee F$ for some finite $F \subseteq D$. A lattice $L$ is called compactly generated iff every element of $L$ is a join of compact elements.

\section{Sharply orthocomplete effect algebras}

In an effect algebra $E$ the set $S(E)=\{x \in E$ $\left.x \wedge x^{\prime}=0\right\}$ of sharp elements plays an important role. In some sense we can say that an effect algebra $E$ is a "smeared set $S(E)$ " of its sharp elements, while unsharp effects are important in studies of unsharp measurements $[4,2]$. S. Gudder proved (see [8]) that, in standard Hilbert space effect algebra $\mathcal{E}(H)$ of bounded operators $A$ on a Hilbert space $H$ between null operator and identity operator, which are endowed with usual + defined iff $A+B$ is in $\mathcal{E}(H)$, the set $S(\mathcal{E}(H))$ of sharp elements forms an orthomodular lattice of projection operators on $H$. Further in [8, Theorem 2.2] it was shown that in every sharply dominating effect algebra the set $S(E)$ is a sub-effect algebra of $E$. Moreover, in [7, Theorem $2.6]$ it is proved that in every S-dominating effect algebra $E$ the set $S(E)$ is an orthomodular lattice. We are going to show that in this case $S(E)$ is bifull in E.

Theorem 1 Let $E$ be an $S$-dominating effect algebra. Then $S(E)$ is bifull in $E$.

Proof. Let $S \subseteq S(E)$.

(1) Assume that $z=\bigvee_{S(E)} S \in S(E)$ exists. Let us show that $z$ is the least upper bound of $S$ in $E$. Let $y \in E$ be an upper bound of $S$. Then $y \wedge z$ exists and it is an upper bound of $S$ as well. Hence, for any $s \in S, s \leq y \wedge z$. As $E$ is sharply dominating, there exists a greatest sharp element $\widetilde{y \wedge z} \leq y \wedge z$ This yields that $s \leq \widetilde{y \wedge z} \leq y \wedge z$, for all $s \in S$, $\widetilde{y \wedge z} \in S(E)$. Hence $z \leq \widetilde{y \wedge} z \leq y \wedge z \leq z$. Then $z=y \wedge z \leq y$ i.e., $z$ is really the least upper bound of $S$ in $E$.

(2) Conversely, let $z=\bigvee_{E} S \in E$ exist. Let $y \in S(E)$ be an upper bound of $S$ in $S(E)$. Then $y \wedge z$ exists and it is again an upper bound of $S$. As in (1) we have that $\widetilde{y \wedge z}$ is the greatest sharp element under $y \wedge z$ and hence $s \leq \widetilde{y \wedge z} \leq y \wedge z \leq z$, for all $s \in S$. This gives that $z=\widetilde{y \wedge z} \in S(E)$. Thus $z=\bigvee_{S(E)} S \in S(E)$.

Corollary 1 If $E$ is a sharply dominating lattice effect algebra then $S(E)$ is bifull in $E$.

Definition 4 An effect algebra $E$ is called sharply orthocomplete (centrally orthocomplete (see [5])) if for any system $\left(x_{\kappa}\right)_{\kappa \in H}$ of elements of $E$ such that there exists an orthogonal system $\left(w_{\kappa}\right)_{\kappa \in H}, w_{\kappa} \in S(E)$ with $x_{\kappa} \leq w_{\kappa}, \kappa \in H$ (an orthogonal system $\left(c_{\kappa}\right)_{\kappa \in H}, c_{\kappa} \in C(E)$ with $x_{\kappa} \leq c_{\kappa}$, $\kappa \in H)$ there exists

$$
\begin{aligned}
& \bigoplus_{\kappa}\left\{x_{\kappa} \mid \kappa \in H\right\}= \\
& \bigvee_{E}\left\{\bigoplus_{E}\left\{x_{\kappa} \mid \kappa \in F\right\} \mid F \subseteq H, F \text { finite }\right\} .
\end{aligned}
$$

Theorem 2 Let $E$ be a sharply orthocomplete $S$ dominating effect algebra. Then

(i) $S(E)$ is a complete orthomodular lattice bifull in $E$.

(ii) $C(E)$ is a complete Boolean algebra bifull in $E$.

(iii) $E$ is centrally dominating and centrally orthocomplete.

(iv) If $C(E)$ is atomic then $\bigvee_{E}\{p \in C(E) \mid p$ atom of $C(E)\}=1$.

Proof. ( $i$ ): From [8, Theorem 2.6] we know that $S(E)$ is an orthomodular lattice and a sub-lattice effect algebra of $E$.

Let us show that $S(E)$ is orthocomplete. Let $S \subseteq S(E), S$ orthogonal. Then for every finite $F \subseteq S$ we have that $\bigoplus_{E} F=\bigvee_{E} F=\bigvee_{S(E)} F \in S(E)$. Moreover, for any $s \in S, s \leq s$. Since $S(E)$ is bifull in $E$ by Theorem 1 and $E$ is sharply orthocomplete we have that $\bigoplus_{E} S=\bigvee_{E} S=\bigvee_{S(E)} S \in S(E)$ exists. Since $S(E)$ is an Archimedean lattice effect algebra we have from [22, Theorem 2.6] that $S(E)$ is complete.

(ii): As $C(E)=\left\{x \in E \mid y=(y \wedge x) \vee\left(y \wedge x^{\prime}\right)\right.$ for every $y \in E\}$, we obtain that $1=x \vee x^{\prime}$ for every $x \in C(E)$ and by the de Morgan Laws $0=x \wedge x^{\prime}$ for every $x \in C(E)$. Hence $C(E) \subseteq S(E)$. It follows by $(i)$ that, for any $Q \subseteq C(E)$, there exists $\bigvee_{S(E)} Q=\bigvee_{E} Q \in C(E)$ because $C(E)$ is full in E, hence $\bigvee_{C(E)} Q=\bigvee_{E} Q$. By the de Morgan Laws there exists $\bigwedge_{E} Q=\left(\bigvee_{E} Q^{\prime}\right)^{\prime}$, where evidently 
$Q^{\prime}=\left\{q^{\prime} \in E \mid q \in Q\right\} \subseteq C(E)$. Hence $\bigwedge_{E} Q \in C(E)$ which gives $\bigwedge_{C(E)} Q=\bigwedge_{E} Q$ (see also $\left.[5]\right)$.

(iii): Let $x \in E$. Using (ii) let us put $c_{x}=\bigwedge_{C(E)}\{c \in$ $C(E) \mid x \leq c\} \in C(E)$. Since $C(E)$ is bifull in $E$ we have that $c_{x}=\bigwedge_{E}\{c \in C(E) \mid x \leq c\}$ (see again [5]).

Since $C(E) \subseteq S(E)$ we immediately obtain that $E$ is centrally orthocomplete.

$(i v)$ : Since $C(E)$ is an atomic Boolean algebra we have $\bigvee_{C(E)}\{p \in C(E) \mid p$ atom of $C(E)\}=1$. As $C(E)$ is bifull in $E$, we have that $\bigvee_{E}\{p \in C(E) \mid p$ atom of $C(E)\}=\bigvee_{C(E)}\{p \in C(E) \mid p$ atom of $C(E)\}=1$.

\section{Sharply orthocomplete lattice effect algebras}

M. Kalina in [12] has shown that even in an Archimedean atomic lattice effect algebra $E$ with atomic center $C(E)$ the join of atoms of $C(E)$ computed in $E$ need not be equal to 1 . Next examples and theorems show connections between sharp orthocompleteness, sharp dominancy and completeness of an effect algebra $E$ as well as bifullness of $S(E), C(E)$ and atomic blocks in a lattice effect algebra $E$. It is worth noting that if $S(E)=\{0,1\}$ then evidently $E$ is $\mathrm{S}$-dominating and sharply orthocomplete.

Example 1 Example of a compactly generated sharply orthocomplete $M V$-effect algebra that is not complete.

It is enough to take the Chang MV-effect algebra $E=\left\{0, a, 2 a, 3 a, \ldots,(3 a)^{\prime},(2 a)^{\prime}, a^{\prime}, 1\right\}$ that is not Archimedean (hence it is not complete). It is compactly generated (every $x \in E$ is compact) and obviously sharply orthocomplete (the center $C(E)=$ $S(E)$ is trivial) and hence sharply dominating.

Example 2 Example of a sharply dominating Archimedean atomic lattice $M V$-effect algebra $E$ with complete and bifull $S(E)$ that is not sharply orthocomplete.

Let $E=\prod\left\{\left\{0_{n}, a_{n}, 1_{n}\right\} \mid n=1,2, \ldots\right\}$ and let

$$
\begin{aligned}
E_{0}= & \left\{\left(x_{n}\right)_{n=1}^{\infty} \in E \mid x_{k}=a_{k}\right. \\
& \text { for at most finitely many } k \in\{1,2, \ldots\}\} .
\end{aligned}
$$

Then $E_{0}$ is a sub-lattice effect algebra of $E$ (hence it is an MV-effect algebra), evidently sharply dominating and it is not sharply orthocomplete (since it is not complete).
$S\left(E_{0}\right)=\prod\left\{\left\{0_{n}, 1_{n}\right\} \mid n=1,2, \ldots\right\}$ is a complete Boolean algebra and $S\left(E_{0}\right)=C\left(E_{0}\right)$ is a bifull sub-lattice of $E_{0}$.

Lemma 1 Let E be a sharply orthocomplete Archimedean atomic $M V$-effect algebra. Then $E$ is complete.

Proof. Let $A \subseteq E$ be a set of all atoms of $E$. Then $1=\bigvee_{E}\left\{n_{a} a \mid a \in A\right\}=\bigoplus_{E}\left\{n_{a} a \mid a \in A\right\}$, $n_{a} a \in C(E)=S(E)$ are atoms of $C(E)$ for all $a \in A$. By [23, Theorem 3.1] we have that $E$ is isomorphic to a subdirect product of the family $\left\{\left[0, n_{a} a\right] \mid a \in A\right\}$. The corresponding lattice effect algebra embedding $\varphi: E \rightarrow \prod\left\{\left[0, n_{a} a\right] \mid a \in A\right\}$ is given by $\varphi(x)=\left(x \wedge n_{a} a\right)_{a \in A}$.

Let us check that $E$ is isomorphic to $\prod\left\{\left[0, n_{a} a\right] \mid\right.$ $a \in A\}$. It is enough to check that $\varphi$ is onto. Let $\left(x_{a}\right)_{a \in A} \in \prod\left\{\left[0, n_{a} a\right] \mid a \in A\right\}$. Then $\left(n_{a}\right)_{a \in A}$ is an orthogonal system and $x_{a}=k_{a} a \leq n_{a} a \in S(E)$ for all $a \in A$. Hence $x=\bigoplus_{E}\left\{x_{a} \mid a \in A\right\}=\bigvee_{E}\left\{k_{a} a \mid\right.$ $a \in A\} \in E$ exists. Evidently, $\varphi(x)=\left(x \wedge n_{a} a\right)_{a \in A}=$ $\left(k_{a} a\right)_{a \in A}=\left(x_{a}\right)_{a \in A}$.

Example 3 Example of a sharply orthocomplete Archimedean MV-effect algebra that is not complete.

If we omit in Lemma 1 the assumption of atomicity in $E$ it is enough to take the MV-effect algebra $E=\{f:[0,1] \rightarrow[0,1] \mid f$ continuous function $\}$, which is a sub-lattice effect algebra of a direct product of copies of the standard MV-effect algebra of real numbers $[0,1]$ that is Archimedean, sharply orthocomplete (the center $C(E)=S(E)=\{0,1\}$ is trivial) and hence sharply dominating. Moreover, $E$ is not complete.

It is well known that an Archimedean lattice effect algebra $E$ is complete if and only if every block of $E$ is complete (see [22, Theorem 2.7]). If moreover $E$ is atomic then $E$ may have atomic as well as non-atomic blocks [1]. K. Mosná [16, Theorem 8] has proved that in this case $E=\bigcup\{M \subseteq E \mid M$ atomic block of $E\}$.

Hence every non-atomic block of $E$ is covered by atomic blocks. Moreover, many properties of Archimedean atomic lattice effect algebras as well as their non-atomic blocks depend on properties of their atomic blocks.

Namely, the center $C(E)$, the compatibility center $B(E)$ and the set $S(E)$ of sharp elements of Archimedean atomic lattice effect algebras $E$ can be expressed by set-theoretical operations on their atomic blocks. As follows, $B(E)=\bigcap\{M \subseteq E \mid$ $M$ atomic block of $E\}, S(E)=\bigcup\{C(M) \mid M \subseteq$ $E, M$ atomic block of $E\}$ and $C(E)=B(E) \cap S(E)$ (see [16]). 
For instance, an Archimedean atomic lattice effect algebra $E$ is sharply dominating iff every atomic block of $E$ is sharply dominating (see [11]). Moreover, we can prove the following:

Theorem 3 Let $E$ be an Archimedean atomic lattice effect algebra. Then the following conditions are equivalent:

(i) $E$ is complete.

(ii) Every atomic block of $E$ is complete.

In this case every block of $E$ is complete.

Proof. $(i) \Longrightarrow(i i)$ : This is trivial, as every block $M$ of $E$ is a full sub-lattice effect algebra of $E$.

$(i i) \Longrightarrow(i)$ : It is enough to show that $E$ is orthocomplete. From [22, Theorem 2.6] we then get that $E$ is complete.

Let $G \subseteq E$ be a $\bigoplus$-orthogonal system. Then, for every $x \in G$, there is a set $A_{x}$ of atoms of $E$ and positive integers $k_{a}, a \in A_{x}$ such that $x=\bigoplus_{E}\left\{k_{a} a \mid\right.$ $\left.a \in A_{x}\right\}$. Moreover, for any $F \subseteq G$ finite we have that $\bigcup\left\{A_{x} \mid x \in F\right\}$ is an orthogonal set of atoms. Hence $A_{G}=\bigcup\left\{A_{x} \mid x \in G\right\}$ is an orthogonal set of atoms of $E$ and there is a maximal orthogonal set $A$ of atoms of $E$ such that $A_{G} \subseteq A$. Therefore there is an atomic block $M$ of $E$ with $A \subseteq M$. By assumption $\bigoplus_{M} G$ exists and $\bigoplus_{M} G=\bigoplus_{E} G$, as $M$ is bifull in $E$ because $E$ is Archimedean and atomic (see [17]).

Theorem 4 Let $E$ be a sharply orthocomplete lattice effect algebra. Then

(i) $S(E)$ is a complete orthomodular lattice bifull in $E$.

(ii) $C(E)$ is a complete Boolean algebra bifull in $E$.

(iii) $E$ is sharply dominating, centrally dominating and S-dominating.

(iv) If moreover $E$ is Archimedean and atomic then $E$ is a complete lattice effect algebra.

Proof. (i), (iii): Let $S \subseteq S(E), S$ be orthogonal. Then, for any $s \in S, s \leq s$. Hence (since $S(E)$ is full in $E) \bigoplus_{E} S=\bigvee_{E} S=\bigvee_{S(E)} S \in S(E)$ exists. Since $S(E)$ is an Archimedean lattice effect algebra we have from [22, Theorem 2.6] that $S(E)$ is complete. Moreover, let $x \in E$ and let $G=\left(w_{\kappa}\right)_{\kappa \in H}$, $w_{\kappa} \in S(E), \kappa \in H$ be a maximal orthogonal system of mutually different elements such that $w_{x}=$ $\bigoplus_{E}\left\{w_{\kappa} \mid \kappa \in H\right\} \leq x$. Let us show that $y \in S(E)$, $y \leq x \Longrightarrow y \leq w_{x} \in S(E)$. Clearly, $w_{x} \in S(E)$. Assume that $y \not \leq w_{x}$. Then $w_{x}<y \vee w_{x} \leq x$. Hence $z=\left(y \vee w_{x}\right) \ominus w_{x} \neq 0$ and $G \cup\{z\}$ is an orthogonal system of mutually different elements such that $y \vee w_{x}=w_{x} \oplus z=\bigoplus_{E}\left\{w_{\kappa} \mid \kappa \in H\right\} \oplus z \leq x$, a contradiction with the maximality of $G$. Therefore $y \leq w_{x}$ and $E$ is sharply dominating, hence Sdominating and from Theorem 2 we get that $E$ is centrally dominating. From Theorem 1, we get that $S(E)$ is bifull in $E$.

(ii): It follows from $(i),($ iii $)$ and Theorem 2.

(iv): Assume now that $E$ is a sharply orthocomplete Archimedean atomic lattice effect algebra. Then every atomic block $M$ of $E$ is a sharply orthocomplete Archimedean atomic MV-effect algebra and hence it is a complete MV-effect algebra by Lemma 1. By Theorem $3, E$ is a complete lattice effect algebra.

Theorem 5 Let $E$ be an atomic lattice effect algebra. Then the following conditions are equivalent:

(i) $E$ is complete.

(ii) $E$ is Archimedean and sharply orthocomplete.

Proof. $\quad(i) \Longrightarrow(i i)$ : By [20, Theorem 3.3] we have that any complete lattice effect algebra is Archimedean. Evidently, any complete lattice effect algebra is sharply orthocomplete.

$(i i) \Longrightarrow(i)$ : It follows from Theorem $4,(i v)$.

\section{Acknowledgement}

The work of the first author was supported by the Slovak Research and Development Agency under contract No. APVV-0375-06 and by the VEGA grant agency, grant number $1 / 0373 / 08$. The second author gratefully acknowledges financial support from the Ministry of Education of the Czech Republic under project MSM0021622409. The third author was supported by the Slovak Research and Development Agency under contract No. APVV-0071-06.

The authors also thank the referee for reading very thoroughly and for improving the presentation of the paper.

\section{References}

[1] Beltrametti, E. G., Cassinelli, G.: The Logic of Quantum Mechanics. Addison-Wesley, Reading, MA, 1981.

[2] Busch, P., Lahti, P. J., Mittelstaedt, P.: The quantum theory of measurement, Lecture Notes in Physics, New Series m: Monographs, Vol. 2, Springer-Verlag, Berlin, 1991.

[3] Chang, C. C.: Algebraic analysis of many valued logics, Trans. Amer. Math. Soc. 88 (1958), 467-490.

[4] Foulis, D. J., Bennett, M. K.: Effect algebras and unsharp quantum logics, Found. Phys. 24 (1994), 1331-1352. 
[5] Foulis, D. J., Pulmannová, S.: Type-Decomposition of an Effect Algebra, Found. Phys.

[6] Greechie, R. J., Foulis, D. J., Pulmannová, S.: The center of an effect algebra, Order 12 (1995), 91-106.

[7] Gudder, S. P.: Sharply dominating effect algebras, Tatra Mt. Math. Publ. 15 (1998), 23-30.

[8] Gudder, S. P.: S-dominating effect algebras, Internat. J. Theoret. Phys. 37 (1998), 915-923.

[9] Jenča, G., Pulmannová, S.: Orthocomplete effect algebras, Proc. Amer. Math. Soc. 131 (2003), 2663-2671.

[10] Jenča, G., Riečanová, Z.: On sharp elements in lattice ordered effect algebras, BUSEFAL $\mathbf{8 0}$ (1999), 24-29.

[11] Kalina, M., Olejček, V., Paseka, J., Riečanová, Z.: Sharply Dominating MV-Effect Algebras, Internat. J. Theoret. Phys.,

DOI: $10.1007 / \mathrm{s} 10773-010-0338-\mathrm{x}$.

[12] Kalina, M.: On central atoms of Archimedean atomic lattice effect algebras, Kybernetika, accepted.

[13] Kalmbach, G.: Orthomodular lattices, Mathematics and its Applications, Vol. 453, Kluwer Academic Publishers, Dordrecht, 1998, 1998.

[14] Kôpka, F.: Compatibility in D-posets, Internat. J. Theoret. Phys. 34 (1995), 1 525-1531.

[15] Kôpka, F., Chovanec, F.: Boolean D-posets, Internat. J. Theoret. Phys. 34 (1995), 1297-1302.

[16] Mosná, K.: Atomic lattice effect algebras and their sub-lattice effect algebras, J. Electrical Engineering 58 (2007), 7/S, 3-6.

[17] Paseka, J., Riečanová, Z.: The inheritance of BDE-property in sharply dominating lattice effect algebras and (o)-continuous states, Soft Comput., DOI: 10.1007/s00500-010-0561-7.
[18] Riečanová, Z.: Compatibility and central elements in effect algebras, Tatra Mt. Math. Publ. 16 (1999), 151-158.

[19] Riečanová, Z.: Subalgebras, intervals and central elements of generalized effect algebras, Internat. J. Theoret. Phys. 38 (1999), 3 209-3220.

[20] Riečanová, Z.: Archimedean and block-finite lattice effect algebras, Demonstratio Mathematica 33 (2000), 443-452.

[21] Riečanová, Z.: Generalization of blocks for $D$ lattices and lattice-ordered effect algebras, Internat. J. Theoret. Phys. 39 (2000), 231-237.

[22] Riečanová, Z.: Orthogonal sets in effect algebras, Demonstratio Math. 34 (2001), 525-532.

[23] Riečanová, Z.: Subdirect decompositions of lattice effect algebras, Internat. J. Theoret. Phys. 42 (2003), 1415-1433.

Doc. RNDr. Martin Kalina, Ph.D.

E-mail: kalina@math.sk

Department of Mathematics

Faculty of Civil Engineering

Slovak University of Technology

Radlinského 11, SK-813 68 Bratislava, Slovakia

Doc. RNDr. Jan Paseka, CSc.

E-mail: paseka@math.muni.cz

Department of Mathematics and Statistics

Faculty of Science

Masaryk University

Kotlářská 2, CZ-611 37 Brno, Czech Republic

Prof. RNDr. Zdenka Riečanová, Ph.D.

E-mail: zdena.riecanova@gmail.com

Department of Mathematics

Faculty of Electrical Engineering and Information Technology

Slovak University of Technology

Ilkovičova 3, SK-812 19 Bratislava, Slovak Republic 